\title{
Urinary Catecholamine Excretion by Various Age Groups with Special Reference to Clinical Value of Measuring Catecholamines in Newborns
}

\author{
TOSHIAKI NAKAI ${ }^{(14)}$ AND RITSUJI YAMADA \\ Department of Clinical Pathology, Dokkyo University School of Medicine, Mibu Town, Tochigi Prefecture, Japan
}

\begin{abstract}
Summary
The sympatho-adrenal system function was assessed in various age groups of normal subjects and in infants with abnormal conditions by assays of daily urinary catecholamine excretion. The daily urinary catecholamine excretion was prone to increase postnatally with time. The group of infants aged 1-5 years exhibited a significantly greater urinary catecholamine excretion than the 0 1-year-age group. No significant difference was observed between the 1-5-year-old and 6-10-year-old age groups in urinary catecholamine excretion. Nor did urinary catecholamine excretion differ significantly between the 11-20 and 6-10-year-old age groups. The values found for daily urinary catecholamine excretion in seven successive age groups of 10-year intervals from 11-80 years showed noticeable inter-subject variation with no significant intergroup differences. These age-related changes in levels of urinary catecholamines from birth to senility indicate a developmental process of the sympatho-adrenal system.

Urinary catecholamine excretions were also measured in infants in special clinical conditions to evaluate the clinical validity of the assay during the neonatal period. The present data indicate that the assay of urinary catecholamine excretion constitutes a useful tool for delineating the clinical features as well as for following up the clinical progress in newborn infants. The present communication is the first to demonstrate the usefulness of urinary catecholamine determination in newborn infants.

Age related changes in levels of urinary catecholamines from birth to senility would indicate developmental process of the sympathoadrenal system. In addition, our results suggest that the measurement of urinary catecholamine excretion acquires a great importance as a parameter for delineating the distressed condition as well as for following up the clinical features of newborn infants.
\end{abstract}

The release of catecholamines from sympathetic nerve endings and adrenal gland is related directly to sympatho-adrenal function. An estimate of catecholamines in the vascular circulation may be an accurate indicator of that sympatho-adrenal function. Only recently have analytic techniques been developed with which to measure circulating norepinephrine and epinephrine at the picomole level of sensitivity $(10,12)$.

These analytic techniques have not found sufficiently widespread application in the determination of circulating catecholamines because first, the use of a radioisotope and second, the cumbersomeness of the assay procedure. Meanwhile, as catecholamines are liberated rather episodically into the circulation, the measurement of the plasma catecholamine level at a given time is of little clinical significance. It is more advisable to determine the catecholamine excretion in 24-h urine specimens, which reflects the daily average level of secretion of these hormones. Assay of catecholamine excretion in $24-\mathrm{h}$ urine specimens, therefore, is adopted at most clinical laboratories as a parameter to assess the sympatho-adrenal function.
Because measurement of elevated catecholamines in the urine is the key to diagnosis of pheochromocytoma, normal values for these parameters in adult subjects have been reported by many investigators $(3,6,8)$. Also, there have been several reports $(4,5$, $11,13)$ of urinary assays for daily catecholamine excretion in successive age groups ranging from the neonatal period to adulthood, with some differences in age-related changes according to the investigators. Determination of urinary catecholamine excretion has undergone widespread application in the diagnosis of pheochromocytoma and neuroblastoma in children and adults. There has been, nevertheless, little work to evaluate its usefulness in neonates. In view of this, the present study was undertaken to explore clinical validity of determination of urinary catecholamine in neonates. Also, age-related changes in urinary catecholamine excretion were determined in urines from birth to senescence to investigate sympatho-adrenal development during life.

\section{MATERIALS AND METHODS}

For the purpose of this study, normal control subjects, ranging in age from 1 day to 80 years, were selected. Among them were 53 babies born in a satisfactory state without an episode of fetal distress. Their mothers had no personal or family history suggestive of endocrine or metabolic disease and their pregnancy was uncomplicated.

Thirty-five infants and school children under 11 years of age were admitted to the study; the infants were selected because no abnormal signs were noted on physical examination and the school children had no significant adverse physical, urinalytic or hematological findings. There were a total of 207 older school children and adults, including 37 elderly subjects over 60 years.

In order to summarize the data the subjects were arbitrarily divided into 11 groups: newborn babies (group 1); 6-month (group 2); 1 through 5 years (group 3); 6 through 10 years (group 4); 11 through 20 years (group 5); 21 through 30 years (group 6); 31 through 40 years (group 7); 41 through 50 years (group 8); 51 through 60 years (group 9); 61 through 70 years (group 10); and 71 through 80 years (group 11).

The urine specimens were collected for $24 \mathrm{~h}$, acidified with hydrochloric acid to $\mathrm{pH} 2-3$ and kept cold. Samples were preserved in a deepfreezer till the time of assay. A number of commonly used drugs and certain beverages, such as $l$-methyldopa, tetracycline, bananas, vanilla and cocoa, which interfere with the fluorimetric estimation, were omitted during the collection period.

In the case of newborn infants, 24-h urine was collected in several successive fractions by means of a series of small urine collection bottles taped onto the skin of the pubic region. Epinephrine and norepinephrine concentrations in the urine were determined by a modification of the fluorometric method $(1,7)$. The method can determine $0.1 \mu \mathrm{g} / 25 \mathrm{ml}$ of epinephrine and norepinephrine as the minimum.

Twenty-four-hour urine specimens were also collected from 
groups of infants in special clinical conditions in order to delineate clinical values for urinary catecholamine excretion in the neonatal period. The groups of newborn infants in special clinical conditions included four clinical conditions: induction of labour by oxytocin, use of forceps, Caesarean section and an Apgar score of $<7$ at 1 min after birth. The group of asphyxial infants was followed by occasional $24-\mathrm{h}$ urine collections along the clinical course. Urine assays for epinephrine and norepinephrine were carried out by the method as in the foregoing cases.

\section{RESULTS}

The results concerning newborn infants are summarized in the Table 1. Results are expressed as the mean \pm S.D. Statistical analyses were performed by non-paired $t$ test. At 1 day after birth, male infants showed mean epinephrine and norepinephrine excretions of $0.5 \pm 0.1$ and $1.8 \pm 0.6 \mu \mathrm{g} /$ day, respectively, and female babies $0.6 \pm 0.1$ and $1.8 \pm 1.0 \mu \mathrm{g} /$ day in $24-\mathrm{h}$ urine. The daily excretion of epinephrine became significantly increased to $0.9 \pm$ $0.2 \mu \mathrm{g} /$ day in males and $0.9 \pm 0.1 \mu \mathrm{g} /$ day in females at $5-7$ days of age, as compared to that observed in $\mathrm{I}$-day-old babies. As can be seen from Table 2 , the average daily urinary epinephrine and norepinephrine excretions of infants and children in Group 3 (15 years) were noted to be significantly higher than those in 6month-old babies, i.e., $1.0 \pm 0.2 \mu \mathrm{g}$ /day of epinephrine and 2.8 $\pm 0.5 \mu \mathrm{g} /$ day of norepinephrine in males and $1.1 \pm 0.2 \mu \mathrm{g} /$ day of epinephrine and $2.4 \pm 0.8 \mu \mathrm{g} /$ day of norepinephrine in females. In Figure 1 the data is summarized concerning daily urinary excretion of catecholamines by male and female subjects in the 11 age-groups, ranging from newborn to 80 years. As noted from the Figure, the 6-10 and 11-20 year groups did not display any significant increase, compared to the 1-5 year group. The mean values for daily urinary catecholamine excretion were higher in males of the 31-40 year group than in males of the 21-30 year and lower age groups, and remained at pratically comparable levels in all the higher age groups up to 80 years, with no significant inter-group differences. In females, the excretion in all the higher age groups were at essentially the same levels as those in the 11-20 years age-group: there were noticeable variabilities in secretion within individual age-groups and hence no significant inter-group difference could be demonstrated.

As shown in Figure 2, the values obtained from the newborn infants in special clinical conditions vary according to the different clinical conditions. The mean daily urinary catecholamine excretion showed higher values in all the subgroups of newborn infants in special clinical conditions than in normal newborn infants. Although the values were apparently higher in groups delivered by Caesarian section and the use of forceps in that order compared with the normal control, they were not statistically significant (Fig. 2). Induction-of-labor-by-oxytocin groups had significant epinephrine excretion over normal level, though, not significant norepinephrine excretion. The increase observed in the group of infants born asphyxiated whose Apgar scores were under 7 was statistically significant over the excretion level in normal newborn infants. Twelve newborn infants had a mean epinephrine excretion level of $0.5 \mu \mathrm{g} /$ day with a S.D. of $0.1 \mu \mathrm{g} /$ day and a mean norepinephrine excretion level of $1.8 \mu \mathrm{g} /$ day with S.D. of $0.8 \mu \mathrm{g} /$ day, whereas the mean epinephrine and norepinephrine level in the urine of asphyxial infants were $2.2 \mu \mathrm{g} /$ day and $4.8 \mu \mathrm{g} /$ day, respectively, with S.D. of $1.0 \mu \mathrm{g} /$ day and $1.3 \mu \mathrm{g} /$ day, (Fig. 2).

Pertinent clinical data from one of the asphyxial infants are summarized in Figure 3: the daily urinary catecholamine excretion, degree of retractions and tachypnea, grading of C-reactive protein test and peripheral blood leukocyte count as clinical signs and dosage of oxygen and digoxin therapy. Treatment included adequate oxygenation without risking the dangers of overoxygenation and intensive digitalization. At 1 day of age, the infant's daily urinary catecholamine excretion was found to be $2.7 \mu \mathrm{g} /$ day

Table 1. Daily urinary catecholamine excretion levels by age in normal newborn infants ( $\mu \mathrm{g} /$ day)

\begin{tabular}{|c|c|c|c|c|}
\hline Days after Birth & \multicolumn{2}{|c|}{ Epinephrine } & \multicolumn{2}{|c|}{ Norepinephrine } \\
\hline 1 & $0.5 \pm 0.1(n=6)$ & $0.6 \pm 0.1(n=6)$ & $1.8 \pm 0.6(n=6)$ & $1.8 \pm 1.0(n=6)$ \\
\hline $4-5$ & $0.7 \pm 0.2(n=6)$ & $0.7 \pm 0.2(n=7)$ & $2.2 \pm 0.6(n=6)$ & $2.2 \pm 0.8(n=7)$ \\
\hline $5-7$ & $0.9 \pm 0.2(n=8)$ & $0.9 \pm 0.1(n=8)$ & $2.0 \pm 0.4(n=8)$ & $2.2 \pm 0.4(n=8)$ \\
\hline
\end{tabular}

Table 2. Daily urinary catecholamine excretion in infants and school children ( $\mu g /$ day)

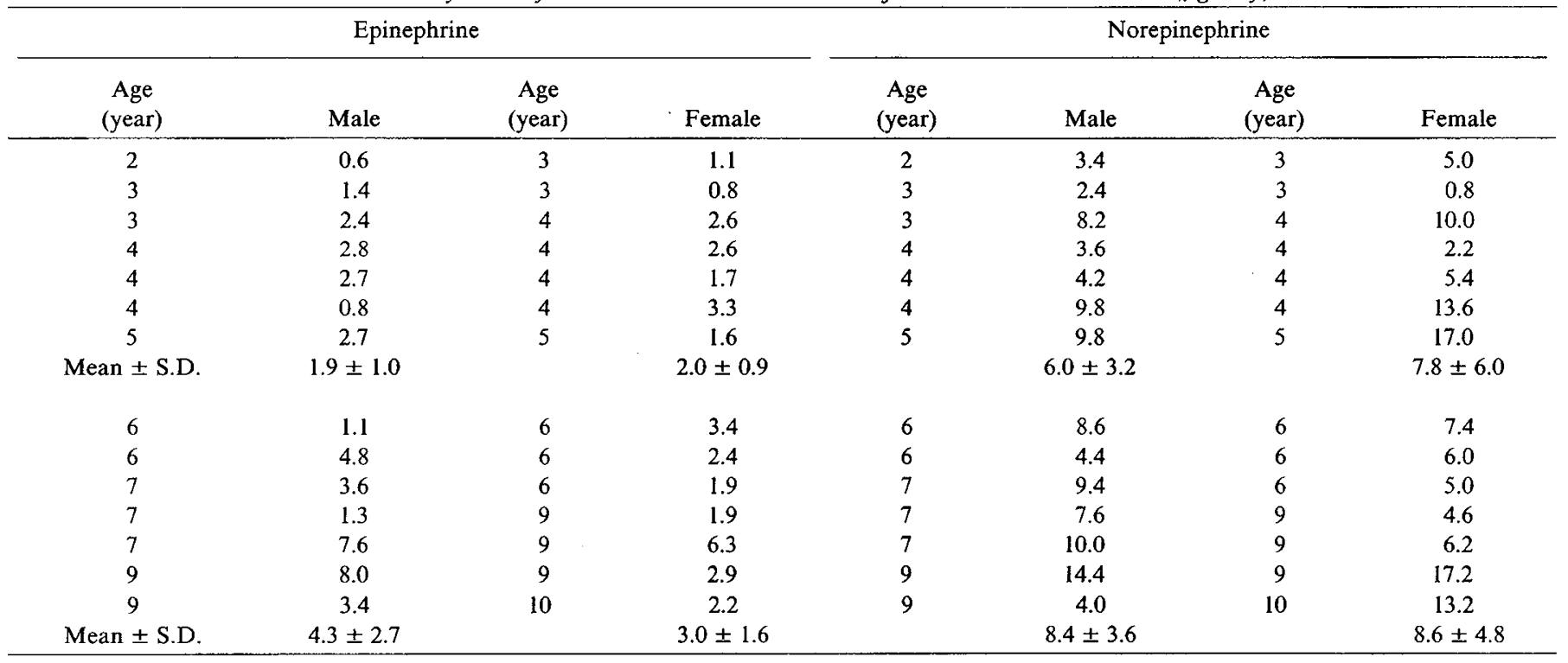




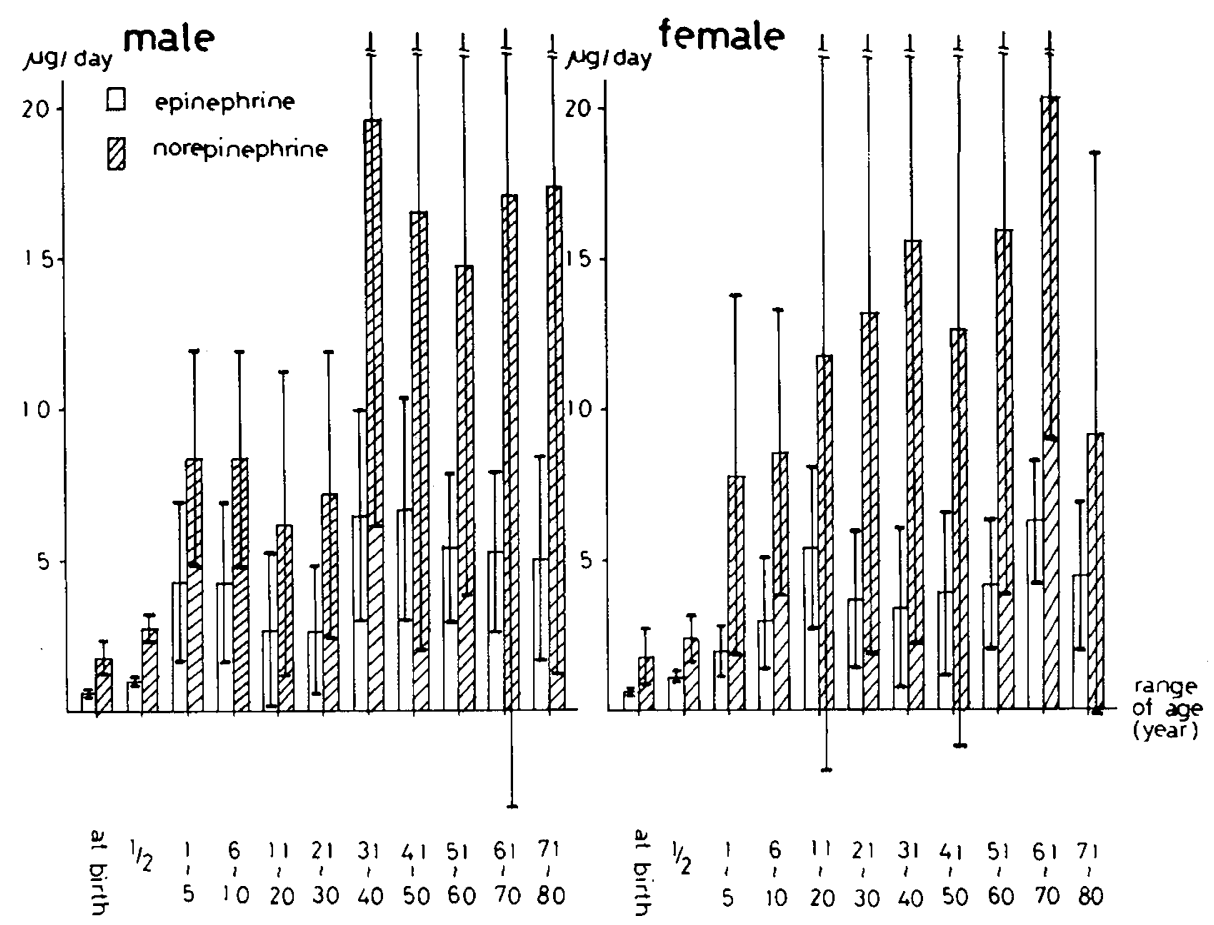

Fig. 1. Daily urinary excretion of catecholamines in various age groups of normal subjects $(\mu \mathrm{g} /$ day $)$. Bars indicate the mean \pm S.D. in each group.

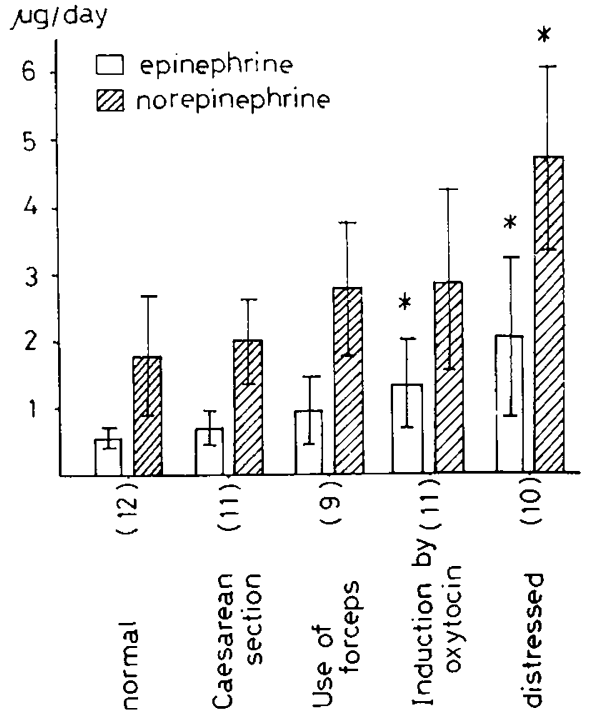

Fig. 2. Urinary catecholamine excretion of newborn infants in special clinical conditions. Bars indicate the mean \pm S.D. in each group. Asterisks show the catecholamine excretion levels were statistically significant $(P$ $<0.05$ ) over normal level.

of epinephrine and $8.5 \mu \mathrm{g} /$ day of norepinephrine and, at 4 days, 1.5 and $6.6 \mu \mathrm{g} /$ day, respectively; thus, remarkably high values for norepinephrine. On the sixth day of life the symptoms began subsiding with a gradual restoration of respiration to normalcy, conversion of the plasma C-reactive protein test to negative and return of peripheral blood leukocyte count and urinary catecholamine excretion to within normal limits. Consequently, the oxygen concentration in oxygen therapy was lowered along with dosage reduction of the cardiotonic was decreased. The data from the remaining asphyxial infants are shown in Table 3. Clinical courses are omitted. In all cases in which elevated urinary catecholamine excretion was demonstrated in the first day of birth, it was found that clinical remission of the distressed condition was generally associated with a decrease in the urinary excretion of catecholamine.

\section{DISCUSSION}

There have been several reports on daily urinary excretion of catecholamines in various age groups of normal subjects. Vorhess (13) has published normal values of daily urinary catecholamine excretion in successive age-groups. According to the report, both the epinephrine and norepinephrine levels in a groups of infants at 1-5 years of age became significantly elevated as compared to a 0-1 year age-group. In a 6-15 year age-group, the norepinephrine excretion was significantly increased. The epinephrine excretion showed a tendency to increase, though not to any significant extent over levels in the 1-5 year age-group. Age-related changes in catecholamines and their metabolites of human urine from birth to adulthood were determined in order to investigate sympatho-adrenal development during life (4). The ratio of vanilmandelic acid/epinephrine + metanephrine + norepinephrine + normetanephrine remains low during a prolonged postnatal period and reaches adult value after 2.5 years of life. According to De Schaepdryver et al. (5), a progressive increase with increasing age for the absolute excretion value of normal children from 1 month to 16 years of age was observed, reaching a maximum in the group of children 6-10 years of age. On the contrary, when the excretion of catecholamines were correlated for surface area, no significant increase were observed from infancy to adulthood (11). Though the use of different methods to quantitate the urinary excretion of catecholamines makes it difficult to compare our results with those previously published, it is safely considered that the maturation process of the sympatho-adrenal system is not achieved in neonate and that it remain low during a prolonged period in childhood reaching full maturation probably near to the fifth year of life.

A previous study reported 3 years age from this department that the plasma concentration in asphyxial infants was found to be significantly elevated as compared to normal infants, suggesting that it might be due to direct effects of hypoxia on the norepinephrine cells (9). Besides the first report of study in man by the present authors, Comline et al. (2) conducted an investigation in domestic animals to clarify the interrelation between the changes 


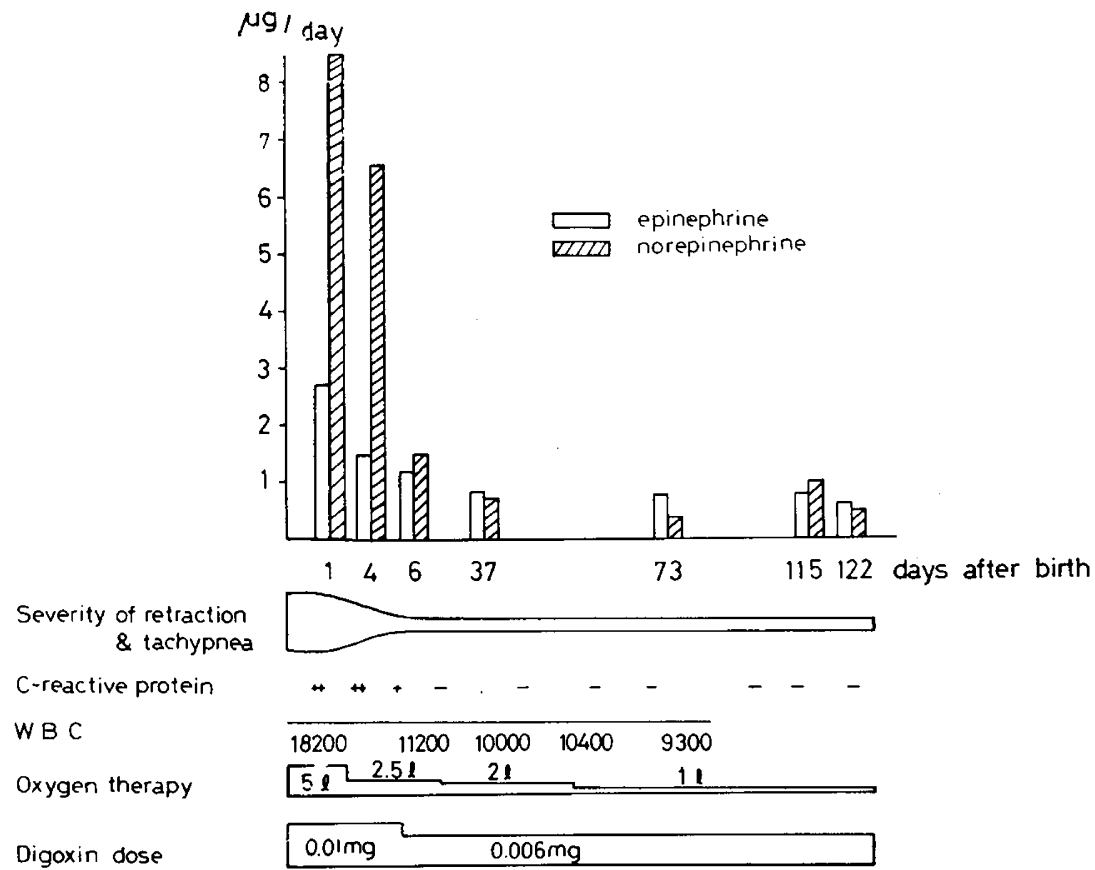

Fig. 3. Changes in urinary catecholamine excretion, clinical course, and therapy in respiratory distress syndrome.

Table 3. Changes in urinary catecholamine excretion in distressed babies ( $\mu g /$ day)

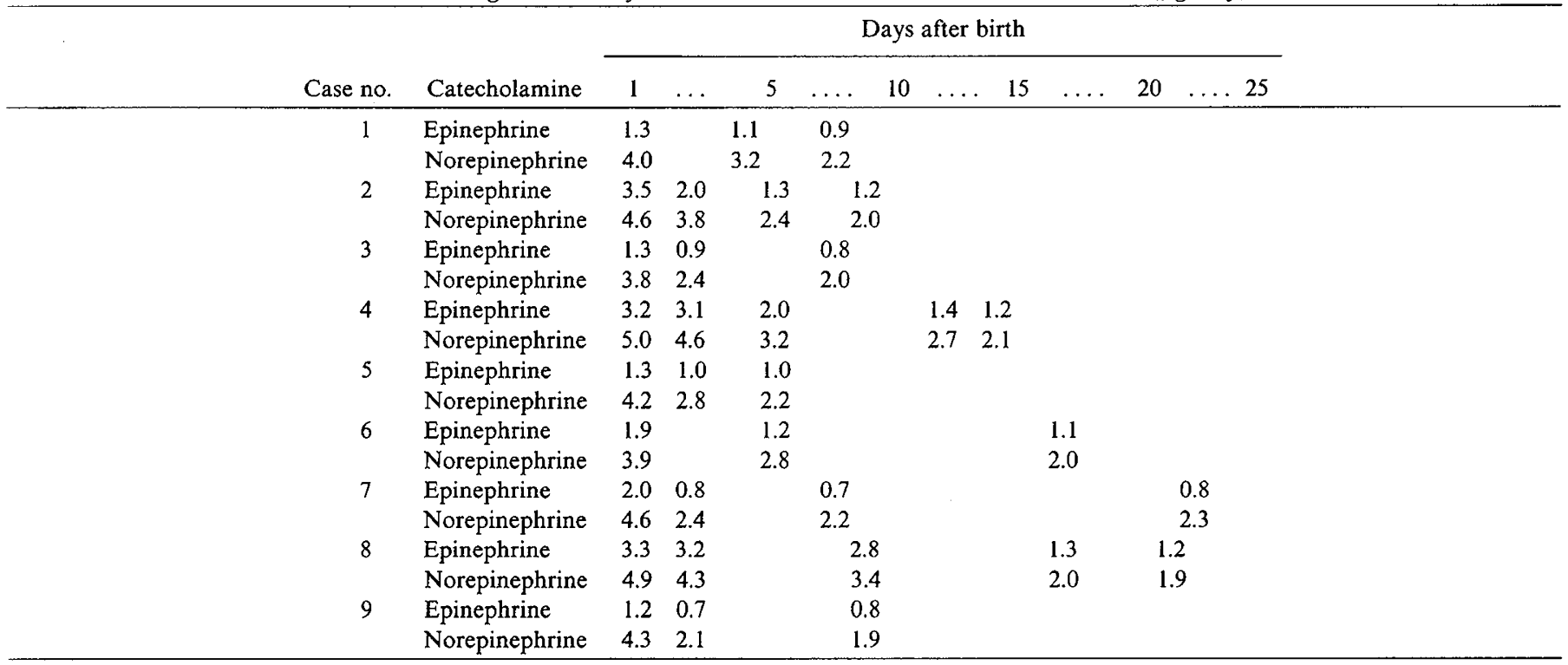

in fetal blood composition and the catecholamine release from the sympatho-adrenal system during perinatal asphyxia, using fetal lambs. Great changes in the fetal blood $\mathrm{Po}_{2}, \mathrm{PCO}_{2}, \mathrm{pH}$ and lactic acid concentration occurred after induction of hypoxia. The adrenal medulla also reacted directly to asphyxia with a secretion, which contained a high proportion of norepinephrine. This high proportion of norepinephrine continued to be secreted throughout the entire period of asphyxia and is the most distinguishing feature of the response of the fetal lamb to asphyxia.

The present study has demonstrated a significantly elevated urinary excretion of both epinephrine and norepinephrine in asphyxiated infants, compared to normal infants, in the first day of birth. In our previous investigation, asphyxial neonates were noted to show slightly but not significantly higher plasma epinephrine levels than normal infants. Whatever the basis for this difference, e.g., metabolic degradation of epinephrine or individ- uality of the subjects studied, it is unquestioned that the concentration of norepinephrine is increased in both the plasma and urine of asphyxial infants. Occasional 24-h urine were obtained through the clinical progress. With subsequent symptomatic amelioration and laboratory parameters to within normal limits, it was found that the daily urinary catecholamine excretion declined to a normal level. The measurement of catecholamine levels in the blood undoubtedly has great clinical validity as a parameter for delineating the distress condition of a newborn infant, as our previous study had demonstrated. Although cord blood specimens can be used for the assay of blood catecholamines promptly after birth, nevertheless, considerable difficulty is inherent in the attempt of blood collection directly from the newborn baby at a few to several days of age. This eventually makes it impracticable to follow the plasma catecholamine levels during the neonatal period to detect and assess abnormalities; hence a remarkable potential 
usefulness of the assay of urinary catecholamine excretion for this purpose as well. The results indicate that it may acquire increasing importance as a useful tool in clinical laboratories.

\section{REFERENCES AND NOTES}

1. Anton, A. H. and Sayre, D.F.: The distribution of dopamine and dopa in various animals and a method for their determination in diverse biological material. J. Pharmacol. Exp. Therp., 145: 326 (1964).

2. Comline, R. S., Silver, I. A., and Silver, M.: Factors responsible for the stimulation of the adrenal medulla during asphyxia in the foetal lamb. J. Physiol., 178: 211 (1965).

3. Crout, J. R., Pisano, J. J., and Sjoerdsma, A.: Urinary excretion of catecholamines and their metabolites in pheochromocytoma. Am. Heart J., 61: 375 (1961)

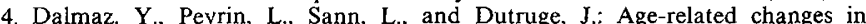
catecholamine metabolites of human urine from birth to adulthood. J. Neural Transmis., 46: 153 (1979).

5. De Schaepdryver, A. F., Hooft, C., Delbeke, M. J., and Van den Noortgaete, M.: Urinary catecholamines and metabolites in children. J. Pediatr., 93: 266 (1978).

6. Drujan, B. D., Sourkes, T. L., Layne, D. S., and Murphy, G. F.: The differential determination of catecholamines in urine. Can. J. Biochem. Physiol., 37: 1153 (1959).

7. Euler, U. S. von and Floding, I.: A fluorimetric micromethod for differential estimation of adrenaline and noradrenaline. Acta Physiol. Scand., 118: 45 (1955).

8. Euler, U. S. von and Floding, I.: Diagnosis of pheochromocytoma by fluorometric estimation of adrenaline and noradrenaline in urine. Scand. J. Clin. Lab., 8: 288 (1956).

9. Nakai, T. and Yamada, R.: The secretion of catecholamines in newborn babies with special reference to fetal distress. J. Perinat. Med., 6: 39 (1978).

10. Nakai, T. and Yamada, R.: Studies on new radioenzymatic assay kit for catecholamine determination and plasma level of catecholamines. (in Japanese) Rinshobyori, 28: 915 (1980).

11. Parra, A., Ramírez del Angel, A., Cervantes, C. and Sánchez, M.: Urinary excretion of catecholamines in healthy subjects in relation to body growth. Acta Endocrinol., 94: 546 (1980).

12. Peuler, J. D. and Johnson, G. A.: Simultaneous single isotope radioenzymatic assay of plasma norepinephrine, epinephrine and dopamine. Life Sci., 21: 625 (1977).

13. Voorhess, M. L.: Urinary catecholamine excretion by healthy children. 1. Daily excretion of dopamine, norepinephrine, epinephrine and 3-methoxy-4-hydroxymandelic acid. Pediatrics, 39: 252 (1967).

14. Requests for reprints should be addressed to: Dr. Toshiaki Nakai, Department of Clinical Pathology, Dokkyo University School of Medicine, Mibu Town, Tochigi Prefecture, Japan.

15. Received for publication May 4, 1982.

16. Accepted for publication October 5, 1982. 